as systems of equations. After that, there is a long chapter on eigenvalue problems. The presentation becomes more condensed in the following chapter devoted to Initial value techniques for boundaryvalue problems.

A special chapter deals with the accuracy of procedures introduced previously. In view of the difficulty of this subject the conclusions here are largely qualitative statements supported by many numerical examples. In the final chapter several topics are touched upon very briefly, such as discontinuous coefficients, Richardson's deferred approach to the limit, collocation, nonlinear eigenvalue problems, etc.

Inasmuch as computation is an art rather than a science it cannot be taught systematically. This book tries to transmit some of the skill, flexibility and flair which a good computer needs in addition to a knowledge of the standard methods. Such a mode of presentation tends to make a book somewhat repetitious and a little exhausting for consecutive reading. This is unavoidable and is not meant as a criticism. Once the reader has become familiar with the basic techniques by studying, say, the first 90 pages, he will find the rest of the book an extremely valuable reference for advice and help, whenever he has to solve boundary value problems for ordinary differential equations.

WOLFGANG WASOW

Problems in Euclidean Space: Application of convexity. By H. G. Eggleston, Pergamon Press, New York 1957. 8+165 pp. \$6.50.

The book is grouped around ten principal, essentially unrelated problems. The author motivates collecting these problems in a book by their implicit or explicit connection with convexity. Actually, there is a much stronger bond between them, namely the spirit of the arguments, which is that of Besicovitch: the problems are special, the proofs require highly ingenious and intricate arguments, but use only the most elementary tools, euclidean geometry and trigonometry besides some simple facts on point sets and measure in $E^{2}$ or $E^{3}$. Most of the work is due to the author, some to others, in particular to Besicovitch.

The first problem concerns the characterization of a nonvoid open set in $E^{2}$ which is the intersection of a decreasing sequence of open connected sets. The second is Ulam's problem, whether a homeomorphism $(x, y) \rightarrow\left(x^{\prime}, y^{\prime}\right)$ of $E^{2}$, or of a square in $E^{2}$, on itself can be approximated by homeomorphisms in which only one variable is changed. Problem 3 deals with sets $E$ of finite linear Hausdorff measure. Denote by $E_{\alpha}$ the projection of $E$ on a line with direction $\alpha$ 
and put $\nu(E)=\inf _{\alpha} H\left(E_{\alpha}\right)$. Then $\nu(E)<2 \pi^{-1} H(E)$ for any measurable $E, \nu(E) \leqq 2^{-1} H(E)$ for connected $E$ and $\nu(E) \leqq H(E) /(\sec \alpha+2 \tan \alpha$ $+\pi-4 \beta-2 \alpha)$, where $\tan \beta=2^{-1} \sec \alpha$ and $2^{-1}+\sin \alpha=4 \cos ^{2} \alpha$ $\cdot\left(1+4 \cos ^{2} \alpha\right)^{-1}$. The proof of these three inequalities takes 37 pages.

Problem 4 is Borsuk's problem in $E^{3}$, whether a set of diameter $\delta$ is always the union of four sets of diameter less than $\delta$. The author prefers his involved solution as possibly generalizable to $n>3$ to Grünbaum's simple solution as a "lucky fluke."

The remaining problems concern bounded closed convex sets in $E^{2}$. Denote by $\delta(X, Y)$ the area of $X \cup Y-X \cap Y$ and put $T(X, n)$ $=\inf \delta\left(X, P_{n}\right)$, where $P_{n}$ traverses all convex polygons with at most $n$ sides. Problem 5 considers several functions of this type, but the principal result is the convexity of $T(X, n)$ as function of $n$.

The other problems are easy to describe briefly: 6. Extremal problems for convex sets solved by triangles, 7 . The asymmetry of sets with constant width, 8 . Sets of constant width contained in a set of given minimal width, 9. Extremal properties of circumscribed triangles, 10 . The closest packing of equilateral triangles.

Clearly, this book will not appeal to some, but will be most delightful to others. In any case, it proves the strength and vitality of plane geometry and should help to revise the attitude of those numerous educators in the U.S.A. who want to all but eliminate plane geometry from high school and college curricula.

\section{Herbert BusemanN}

Die Lehre von den Kettenbruchen. Vol. II. Analytisch-funktionentheoretische Kettenbruche. By Oskar Perron. 3d ed. Stuttgart, Teubner, 1957. $6+316$ pp. DM 49.

Earlier editions of this text on continued fractions contained in a single volume a part devoted to the arithmetic theory and a part devoted to function-theoretic aspects (analytic theory). In the third edition these two parts have been published in separate volumes. Volume I was published in 1954 and the publication of the present book, Volume II, marks the completion of the third edition. Because the second edition is well known and has served as a standard reference since its appearance in 1929 , only the major changes occurring in Volume II are noted below.

Chapter I includes new sections on continued fractions with prescribed approximants ( $\$ 3$ ) and on a formula of Ramanujan ( $\$ 8$ ). Chapter II contains additional sections on new convergence critieria, including the parabola theorem, $(\$ 17)$, and on the convergence of the Ramanujan continued fraction (\$16). Several theorems on conver- 